\title{
Similitudes et différences politiques et économiques entre les deux états roumains
}

\author{
Vasile Maxim¹, Oleg Buga², Sandu Mitreanu ${ }^{3}$ \\ ${ }^{1}$ Ion Creangă Pedagogical State University, Republic of Moldova. \\ 2 Alecu Russo State University of Bălți, Republic of Moldova. \\ ${ }^{3}$ Babeș-Bolyai University of Cluj-Napoca, Romania.
}

To cite this article: Maxim, V., Buga, O. \& Mitreanu, S. (2017). Similitudes et différences politiques et économiques entre les deux états roumains. Lucrările Seminarului Geografic Dimitrie Cantemir, Vol. 45, pp. 175-178. DOI: 10.15551/lsgdc.v45i0.14

To link to this article: http://dx.doi.org/10.15551/lsgdc.v45i0.14 


\title{
SIMILITUDES ET DIFFERENCES POLITIQUES ET ECONOMIQUES ENTRE LES DEUX ETATS ROUMAINS
}

\author{
Vasile Maxim ${ }^{1}$, Oleg Buga $^{2}$, Sandu Mitreanu ${ }^{3}$
}

\begin{abstract}
This paper aims to highlight the most important similarities and discrepancies within the Romanian space through the homogeneity of demographic change, especially in Bessarabia. Similar patterns in economic development are analyzed with the presence of similar political factors in the postwar period to the present. The most important processes of the political and economic environment in both countries are specified.
\end{abstract}

Mots clés: similitudes, divergences, homogénéité, modèle économique, infrastructure, expansion, conséquences politiques, protectionnisme, investissements

\section{Introduction}

L'intérêt politique pour le territoire roumain apparaît quand l'espace géographique cade se retrouve sous l'influence de trois empires : austro-hongrois, tsariste et ottoman.

Friedrich Ratzel, le fondateur de la Géographie Politique affirme que l'apparition de l'état roumain moderne en 1859 est due au conflit entre la Russie et la Turquie. L'homogénéité de l'espace géographique roumain sous l'aspect géographique est déformée par la politique de ces empires, qui dans le dernier moment enregistre des répercussions envers l'environnement socio - économique. Comme un exemple éloquent sert l'évolution démographique de la Bessarabie après son annexion à l'Epire Tsariste en 1812 .

De cette manière, la population de la Bessarabie en 1810 atteint plus de 327 milles d'habitants d'où 95\% étaient Roumains. En 1817 sont enregistrés plus de 420 milles d'habitants, la population roumaine représente $86 \%$. En 1865 (selon les données de Ion Nistor) les Roumains constituent 74 \%. Le recensement du 1897, effectué par l'administration tsariste montre que seulement $47,6 \%$ sont des Roumains autochtones, 19,6\% sont Ukrainiens, $11 \%$ sont Juifs, $8 \%$ sont Russes, 5,3\% sont Bulgares, 3,1\% sont Allemands, 2,9\% sont Gagaouzes.

\footnotetext{
${ }^{1}$ Universitatea Pedagogică de Stat "Ion Creangă” din Chișinău, Facultatea Istorie şi Geografie, maxivali@yahoo.com

2 Universitatea de Stat Alecu Russo din Bălți bugaoleg26@mail.ru

3 Universitatea Babeș - Bolyai Cluj - Napoca alex_mitreanu@yahoo.com
} 


\section{L'expansionnisme russe par rapport à l'espace géographique roumain}

Par l'annexion de la Bessarabie, la Russie Tsariste essaye de créer des écarts d'ordre ethnique par l'acceptation en masse des personnes colonisées étrangères, pour que la population autochtone devienne minoritaire. La russification en masse provoque la migration de la population autochtone à l'autre côté de la rivière Prut. La construction des chemins de fer sur le territoire de la Bessarabie arrive à accélérer le flux d'entrée de la population russe, surtout après 1860. Le territoire est dominé par trois chemins de fer: celui central (Tiraspol Tighina - Chişinău - Ungheni - Iaşi, ayant l'objectif de créer une liaison avec l'espace balkanique), celui de sud - ouest (Tighina-Galaţi) construit dans la période de la guerre russoturque du 1877, qui en fait avait le but d'assurer l'accès rapide des troupes armées à l'embouchure du Danube et celui du nord (construit en 1882) qui facilitait la connexion avec la jonction ferroviaire de Râbniţa - Rezina - Bălţi afin de pénétrer sur le territoire de la Bucovine, qui était sous l'influence de l'Autriche.

Dès le début, sont enregistrées des divergences à l'écartement des magistrales existantes (1435 $\mathrm{mm}$ et $1525 \mathrm{~mm}$ ) dans l'espace roumain, fait qui arrive à retenir les flux de transport de marchandises et de passagers.

L'unification des territoires roumains en 1918 a modifié la situation géographique de la Bessarabie en faveur de la population autochtone. De la même façon, les chemins de fer suivent les paramètres roumains pour la moitié d'une année, fait qui a stimulé le flux d'entrée des marchandises et des passagers sans la présence d'aucun obstacle d'ordre technique.

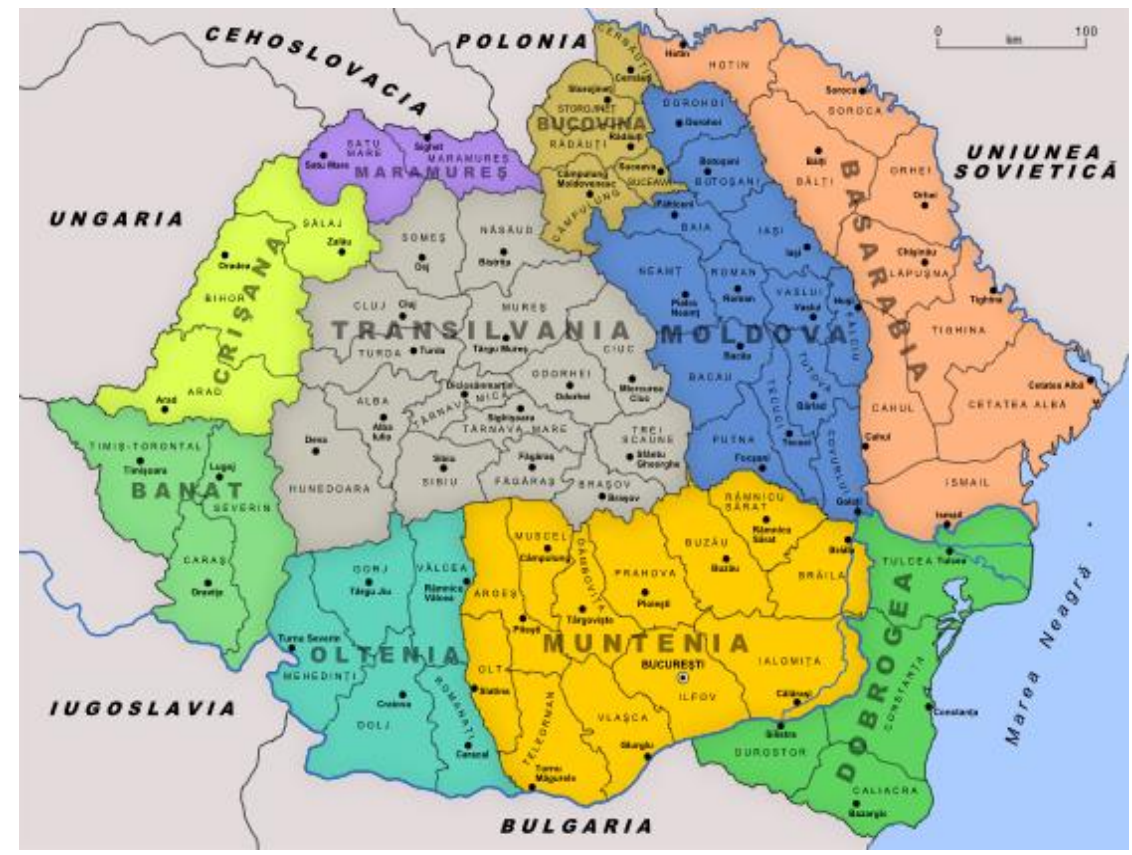

Figure 1. La Roumanie dans la période inter guerre 


\section{Les conséquences, les similitudes et les différences politiques et économiques}

L'accord Ribbentrop - Molotov a des effets négatifs, provoqués par les atrocités commises du régime soviétique sur le territoire de la Bessarabie: les déportations en masse, le fait de renoncer à quelques territoires en faveur de l'Ukraine, la création de la République Soviétique Socialiste Moldave, l'apparition de l'alphabet cyrillique, la tentative de créer une nation artificielle soviétique moldave afin de démontrer la différence envers la population de l'autre côté du Prut, la russification en masse de l'environnement socio-économique et culturel par l'acceptation de la population d'origine slave au milieu de cet espace.

Les conséquences susmentionnées approfondissent les divergences politiques et socio - économiques entre la Roumanie et la République Soviétique Socialiste Moldave.

L'interdiction des échanges culturels entre la Roumanie et la République Soviétique Socialiste Moldave (l'absence totale de la presse et la littérature roumaine sur le territoire de la RSSM). Les similitudes économiques et politiques sont caractérisées par l'acceptation du modèle soviétique d'industrialisation de la Roumanie et la collectivisation du secteur agricole. Les répressions politiques soviétiques influencent celles de la Roumanie. Un exemple éloquent est la construction du canal Danube - Mer Noire.

L'économie de ces deux états a connu des modifications qualitatives et quantitatives, avec la disparition du système économique socialiste au début des années 90 du siècle XX. Leur industrialisation a été réalisée selon le modèle soviétique en l'absence de la propriété privée.

L'économie hypercentralisée des deux états connaît une crise profonde par les déséquilibres existants au niveau économique de l'espace post-communiste.

$\mathrm{Au}$ niveau macroéconomique, la majorité des entreprises n'ont pas été protégées des facteurs internes et externes, de manière à éviter le phénomène de dumping, qui finalement, a favorisé la faillite des entreprises de la Roumanie, ainsi que de celles de la République de Moldavie.

Des exemples pertinents peut être tirés de l'industrie des automobiles des deux états, par exemple :' Tractorul Braşov "' ou l'Usine des téléviseurs ' ALFA' de Chişinău.

Le manque des politiques protectionnistes des deux états, ainsi que le rejet des investissements directs étrangers des agents économiques nationaux, qui ont provoqué la crise économique à travers la faillite et la disparition de certaines branches industrielles avec des répercussions négatives sur la branche agricole.

L'environnement politique des deux états a conduit vers la crise économique, due à l'acceptation aveugle des conditions de financement par les structures internationales, qui dans la majorité des cas étaient au détriment des intérêts nationaux.

De cette façon, la Roumanie, qui vers la fin des années 80 n'avait pas des dettes extérieures, en enregistrant une balance commerciale et financière excédentaire, est devenue prochainement dépendante du FMI, qui jusqu'au présent promeut une politique qui mène à l'aggravation de la qualité de la vie, en imposant des politiques de privatisation de certains objets économiques, considérés d'intérêt stratégique national.

Dans les deux états, les procès de libéralisation de l'environnement économique se sont déroulés dans le détriment de l'environnement social par la réduction de la qualité de la vie et l'augmentation du taux de chômage en l'absence des opportunités de stimulation de la croissance économique. 
Les intérêts socio-économiques à court terme se réalisent jusqu'à présent dans les deux états seulement pour les exposants des partis politiques du Gouvernement.

Le fléau de la corruption est caractéristique pour la majorité des partis politiques des deux pays.

L'exode massif de la population apte de travail est omniprésent dans les deux états à partir du milieu des années '90, fait qui a créé le phénomène paradoxal, quand l'économie et la stabilité financière sont étroitement dépendantes des rémittences de l'extérieur, même à court terme.

Une des plus grandes et importantes divergences économiques par rapport à la République de la Moldavie est enregistrée en ce qui concerne la qualité de l'infrastructure des réseaux de transport de la Roumanie (par l'allocation et l'utilisation des fonds européens), qui en effet servent comme substrat de base pour le développement d'une économie moderne.

Un exemple digne de comparaison est l'indice PIB de la Roumanie qui dépasse 177 milliards de dollars américains, le PIB par habitant est 14 milles, tandis que le PIB de la République de Moldavie est seulement de 6 milliards USD et atteint 3 milles dollars par habitants.

\section{Conclusions}

Les divergences d'ordre politique sont enregistrées par le niveau élevé de la corruption des personnes politiques de la République de la Moldavie (le vol et le blanchiment des milliards de dollars au sein des banques nationales).

Seulement une éventuelle réunification politique de ces deux états peut produire des changements d'ordre qualitatif et quantitatif par la réduction des divergences politiques et économiques en vue de constituer un marché économique viable avec des effets bénéfiques durables pour la population roumaine des deux côtés du Prut.

\section{Bibliographie :}

1. Maxim V. Model de Integrare a Republicii Moldova în cadrul UE - aspecte geopolitice, În Materialele simpozionului jubiliar internațional "Mediul și dezvoltarea durabilă" (pag.249.) Ed. Labirint Chișinău 2009.

2. Maxim V. Unele aspecte geopolitice legate de infrastructura căilor de transport din Basarabia, $\hat{I} n$ Politica Marilor Puteri în Balcani și Europa Centrală. Materialele simpozionului internațional din cadrul UPS “Ion Creangă.” Chișinău 2014. 